\title{
Qualidade físico-química e sensorial da carne de peito de matrizes pesadas de descarte
}

\author{
Physical, chemical and sensorial breast meat quality of spent breeder hens
}

\author{
Claudia Marie Komiyama ${ }^{\mathrm{I} *}$ Ariel Antonio Mendes $^{\mathrm{I}}$ Cristiane Sanfelice $^{\mathrm{I}}$ Marleide Costa Cañizares ${ }^{\mathrm{I}}$ \\ Roberto de Oliveira Roça ${ }^{I I}$ Sabrina Endo Takahashi' ${ }^{I}$ Luciana Rodrigues $^{\mathrm{I}}$ \\ Gil Ignácio Lara Cañizares ${ }^{I}$ Ibiara Correia de Lima Almeida Paz \\ Karen Franco de Godoi Cardoso
}

\section{RESUMO}

O objetivo do presente trabalho foi avaliar as características de qualidade: $\mathrm{pH}$, cor, valor $\mathrm{R}$, perda por exsudação, capacidade de retenção e absorção de água, capacidade de emulsificação, perdas por cocção, força de cisalhamento e análise sensorial da carne de matrizes pesadas de descarte de frangos de corte. A carne de peito de matrizes apresentou valores médios do parâmetro $p H$, valor $R$, perda por exsudação e valor de $L^{*}$ de 5,70, 1,43, 2,00 e 50,11, respectivamente. Para a capacidade de retenção e absorção de água, perda de peso por cozimento e força de cisalhamento, os valores médios foram de 77, 58, $18 \%$ e 4,94kgf $\mathrm{cm}^{-2}$, respectivamente. $\mathrm{Na}$ análise sensorial, a carne de matriz apresentou baixa intensidade de maciez $(6,9)$ e menor suculência $(3,4)$ e foi a mais elástica, borrachenta e difícil de deglutir. A carne de matrizes pesadas de descarte apresenta boas características de qualidade tecnológicas que possibilitam sua utilização como matéria-prima para a elaboração de industrializados.

Palavras-chave: qualidade da carne, maciez, matrizes, frangos de corte.

\section{ABSTRACT}

The aim of this research was evaluate the meat quality broiler breeder hens: $p H$, color, $R$ value, drip loss, water holding capacity, water absorption capacity, emulsion capacity, cooking loss, shear force and sensorial analysis. The breast meat of broiler hens presents means values for $\mathrm{pH}, \mathrm{R}$ value, drip loss and $L^{*}$ value parameter of 5.70, $1.43,2.00$ and 50.11, respectively. The means value for water retention capacity, water absorption capacity, cooking loss and shear force, were 77, 58, 18\% e 4.94kgf $\mathrm{cm}^{-2}$ respectively. For sensory analysis, the meat of broiler hen present lower tenderness intensity (6.9), lower succulence (3.4), more elasticity, rubbery and swallowing difficulties. The meat of broiler hens presented good technology quality characteristics that make it possible to be used as meat for elaborated industry.

Key words: meat quality, tenderness, broiler breeder, broiler.

\section{INTRODUÇÃO}

As matrizes pesadas concentram suas características na produção de pintos comerciais de grande vigor híbrido. São aves grandes, pesando de 3,0 a 4,0kg, com a conformação que se espera de um frango, ou seja, muita carne no peito e nas coxas. Porém, apresentam grande deposição de gordura tanto subcutânea, quanto abdominal (NUNES, 2003). No entanto, essas aves tornam-se disponíveis ao abate ao final de seu ciclo produtivo, podendo ser comercializadas pela indústria avícola.

Segundo dados da União Brasileira de Avicultura (UBA), o alojamento de matrizes de corte em 2008 foi acima das previsões iniciais, sendo alojadas

'Departamento de Produção Animal, Faculdade de Medicina Veterinária e Zootecnia (FMVZ), Universidade Estadual Paulista (UNESP), campus de Botucatu, 18618-970, São Paulo, SP, Brasil. E-mail: claudiakomiyama@yahoo.com.br. *Autor para correspondência.

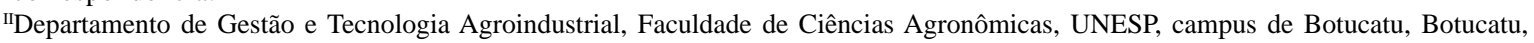
SP, Brasil. 
48.564.097 matrizes, o que representa um crescimento inédito de $14,3 \%$ em relação ao ano de 2007, que apresentou um alojamento de 42.481 .788 matrizes (UBA, 2009). Aliado ao crescente número de matrizes alojadas a cada ano, está em trâmite o Plano de Regionalização Sanitária, que segue as disposições do Capítulo 1.3.5. do Código Sanitário para os animais terrestres da OIE, em que é previsto o controle do tráfego de animais, com o objetivo de preservar o mercado internacional e suas restrições às importações de produtos de origem animal (OIE, 2002). Dessa forma, cada Estado deve dar um destino adequado para as suas matrizes de descarte. Assim, uma alternativa viável seria o abate dessas aves em abatedouros comerciais quando estas chegam ao final de seu ciclo de postura. Portanto, existe a necessidade do conhecimento das características de qualidade dessa carne para que esta possa ser aproveitada de forma a aumentar os lucros da indústria avícola.

Nesse sentido, informações sobre as propriedades físico-químicas e funcionais, bem como se essas propriedades determinam a qualidade do produto final, são essenciais para a obtenção de produtos de qualidade. Um dos maiores problemas com relação a essa carne é a perda da maciez. NAKAMURA et al. (1975) relataram que a dureza da carne de aves de descarte é causada pela alta quantidade de colágeno termo estável, e essa dureza tem impedido seu uso em alimentos cárneos e reduzido seu valor monetário nos Estados Unidos (NURMAHMUDI \& SAMS, 1997).

Assim, os objetivos do presente trabalho foram avaliar as características de qualidade da carne de peito de matriz pesada em final de ciclo produtivo, verificando se esta pode ser comercializada in natura e avaliar também suas propriedades tecnológicas para a sua utilização como matéria-prima em produtos industrializados.

\section{MATERIAL E MÉTODOS}

As amostras de carne de peito de matrizes de descarte foram obtidas em linha comercial de abate, na cidade de Itapetininga, São Paulo (SP). Para evitar possíveis interferências no manejo ante-mortem, foram coletadas 120 amostras de filés de peito desossado (Pectoralis major), divididas em seis coletas de 20 amostras cada. Com 1,5 e quatro horas após o abate, foram realizadas as análises de $\mathrm{pH}$ e valor $\mathrm{R}$ de todas as amostras. Em seguida, as amostras foram transportadas para o laboratório de qualidade de carne da FMVZ/UNESP, campus de Botucatu, onde foram avaliados os seguintes parâmetros com 24 horas postmortem: $\mathrm{pH}$, valor $\mathrm{R}$, cor objetiva, perda por exsudação (drip loss), capacidade de retenção e absorção de água, capacidade de emulsificação, perdas por cocção e força de cisalhamento.

$\mathrm{O} \mathrm{pH}$ foi mensurado segundo BENDALL (1973), sendo utilizada solução tamponada de iodoacetato de sódio $5 \mathrm{mM} \mathrm{KCl}^{-1} 150 \mathrm{mM}$ e realizada a leitura do $\mathrm{pH}$ com pHmetro (Hommis, modelo 238) acoplado a uma sonda (Digimed, modelo CF1). O valor R foi obtido segundo HONIKEL \& FISHER (1977). A cor objetiva foi determinada por meio do colorímetro Konica Minolta, modelo CR-400, no sistema CIELab, em que foram avaliados os parâmetros $L^{*}$ (luminosidade), a* (teor de vermelho) e b* (teor de amarelo) (VAN LAACK et al., 2000).

A capacidade de absorção de água foi determinada a partir da pesagem de $30 \mathrm{~g}$ de carne moída, com a adição de $90 \mathrm{~mL}$ de água destilada. Foram pesados $35 \mathrm{~g}$ da pasta obtida e centrifugados por 15 minutos a 3000rpm. O sobrenadante foi desprezado, o tubo foi pesado e a capacidade de absorção foi calculada da seguinte forma: $\% C A A=\{[(\mathrm{PP}-\mathrm{PC})-\mathrm{PS}] / \mathrm{PC}\} * 100$, em que: $\mathrm{PP}=$ peso da pasta, $\mathrm{PC}=$ peso da carne na pasta e PS = peso do sobrenadante (ROÇA, 1986). Para a análise de perda por exsudação (drip loss), uma amostra de $100 \mathrm{~g}$ de carne foi suspensa em rede e colocada em saco plástico inflado, por período de 48 horas, com temperatura de $2^{\circ} \mathrm{C}$, e foi calculada a porcentagem de perda de água (RASMUSSEN \& ANDERSON, 1996). A capacidade de retenção de água foi realizada segundo a metodologia descrita por HAMM (1960), baseada na medição da perda de água liberada quando aplicada uma pressão $\left(10 \mathrm{~kg} 5 \mathrm{~min}^{-1}\right)$ sobre o tecido muscular.

Para a capacidade de emulsificação, foram pesadas $25 \mathrm{~g}$ da amostra e homogeneizadas em 100mL de solução de $\mathrm{NaCl} 1 \mathrm{M}$. Em um béquer, foram pesados 2,5g dessa solução inicial e foram adicionados $20 \mathrm{~mL}$ de água destilada e 10ml de óleo de soja. Em seguida, ocorreu o emulsionamento e então foi iniciada a adição de óleo até a quebra da emulsão (SWIFT et al., 1961 e modificada por NUNES, 2003). Para a análise de perda de peso por cozimento, as amostras foram cozidas em chapa metálica aquecida até a temperatura interna do filé atingir $82^{\circ} \mathrm{C}$ e, por diferença de peso antes e após o cozimento, se obteve a perda de peso por cozimento (HONIKEL, 1987). Para determinação da força de cisalhamento, foram utilizadas as amostras usadas para determinação da perda de peso por cozimento, as quais foram cortadas em paralelepípedos com dimensões de $1 \times 1 \times 2 \mathrm{~cm}$ e colocadas com as fibras orientadas no sentido perpendicular às lâminas do acessório WarnerBlatzler acoplado ao texturômetro TA.XT plus (Stable Micro Systems) (AMSA, 1995). 
As amostras para determinação das características sensoriais foram obtidas no mesmo abatedouro, sendo realizada uma única coleta de 20 peitos de matriz de descarte e 20 peitos de frangos de corte que foi amostrada para a avaliação microbiológica (Coliformes fecais e Mesófilos), segundo SPLITTSTOESSER (1992). A avaliação sensorial utilizada foi a Análise descritiva quantitativa (ADQ), utilizando amostras codificadas com algarismos de três dígitos, juntamente com as fichas geradas com os termos descritores, em cabines individuais, no Laboratório de Análise Sensorial do Departamento de Gestão e Tecnologia Agroindustrial/Unesp. Essa análise foi conduzida conforme ROÇA et al. (1988), com oito provadores treinados e selecionados (ROÇA \& BONASSI, 1985) em três dias consecutivos. Foram realizadas avaliações de aroma, sabor, maciez, suculência e mastigabilidade por meio de escala não estruturada de nove pontos. As amostras foram submetidas à salga com salmoura a $10 \%$, por 20 minutos, e submetidas ao aquecimento em chapa elétrica com dupla resistência, regulada para $200^{\circ} \mathrm{C}$, até a temperatura interna atingir $85^{\circ} \mathrm{C}$, e servidas imediatamente aos provadores. Este trabalho foi submetido e aprovado pela Câmara de Ética em Experimentação Animal e pelo Comitê de Ética para uso de Seres Humanos, tendo sido realizado de acordo com os princípios éticos na experimentação animal e o uso de seres humanos.

Os resultados foram submetidos ao programa estatístico SAS, sendo utilizados a estatística descritiva, a Análise de Variância (ANOVA) e o teste de comparação de médias de Tukey (SAS, 1998).

\section{RESULTADOS E DISCUSSÃO}

A tabela 1 mostra os resultados dos valores médios de $\mathrm{pH}$ e valor $\mathrm{R}$ do peito de matriz analisados em três tempos (1,5; quatro e 24 horas post-mortem) coletados em seis visitas diferentes. Foram observadas diferenças significativas $(\mathrm{P} \leq 0,05)$ para o $\mathrm{pH}$ entre as diferentes coletas, e na coleta 4 foram observadas as maiores médias de $\mathrm{pH}(6,15)$ quando comparadas com as demais coletas. Isto pode ter ocorrido, pois em cada coleta foi utilizada carne provenientes de aves de lotes diferentes que sofreram diferentes tratamentos no antemortem, como, por exemplo, tempo de jejum, distância de transporte e tempo de espera no abatedouro, etapas que não puderam ser controladas para a realização deste trabalho. Segundo alguns autores, essas etapas ante-mortem podem afetar a qualidade da carne de aves pelo estresse produzido, levando à alteração no $\mathrm{pH}$ da carne (TAKAHASHI, 2007; KOMIYAMA, 2006, KOMIYAMA et al. 2008).
Tabela 1 - Avaliação do $\mathrm{pH}$ e valor $\mathrm{R}$ da carne de peito de matrizes pesadas de descarte realizado em três tempos (uma, cinco, quatro e 24 horas post-mortem) e em seis coletas ( 1 a 6$)$.

\begin{tabular}{|c|c|c|c|}
\hline Parâmetros & $\mathrm{pH}$ & & Valor R \\
\hline \multicolumn{4}{|l|}{ Coletas } \\
\hline 1 & \multicolumn{2}{|l|}{$6,00 \mathrm{BC}$} & 1,30 \\
\hline 2 & \multicolumn{2}{|l|}{$5,98 \mathrm{C}$} & 1,24 \\
\hline 3 & \multicolumn{2}{|l|}{$6,11 \mathrm{AB}$} & 1,39 \\
\hline 4 & \multicolumn{2}{|l|}{$6,15 \mathrm{~A}$} & 1,25 \\
\hline 5 & \multicolumn{2}{|l|}{$5,93 \mathrm{C}$} & 1,25 \\
\hline 6 & \multicolumn{2}{|l|}{$6,02 \mathrm{BC}$} & 1,23 \\
\hline \multicolumn{4}{|l|}{ Tempo } \\
\hline 1,5 & \multicolumn{2}{|l|}{$6,60 \mathrm{~A}$} & 1,07A \\
\hline 4 horas & \multicolumn{2}{|l|}{$5,79 \mathrm{~B}$} & $1,29 \mathrm{~B}$ \\
\hline 24 horas & \multicolumn{2}{|l|}{$5,70 \mathrm{C}$} & $1,43 \mathrm{C}$ \\
\hline \multicolumn{4}{|l|}{ Probabilidade } \\
\hline Coleta & \multicolumn{2}{|l|}{$<0,0001$} & 0,0972 \\
\hline Tempo & \multicolumn{2}{|l|}{$<0,0001$} & $<0,0001$ \\
\hline Coleta x Tempo & \multicolumn{2}{|l|}{0,0034} & 0,2346 \\
\hline \multicolumn{4}{|c|}{ Desdobramento pH } \\
\hline & \multicolumn{3}{|c|}{ Tempo (horas post mortem) } \\
\hline Coleta & 1,5 horas & 4 horas & 24 horas \\
\hline 1 & 6,62 & 5,74 & 5,65 \\
\hline 2 & $6,55 a$ & $5,68 b$ & $5,71 b$ \\
\hline 3 & 6,73 & 5,90 & 5,69 \\
\hline 4 & $6,74 a$ & $5,86 b$ & $5,85 b$ \\
\hline 5 & $6,35 a$ & $5,74 b$ & $5,70 \mathrm{~b}$ \\
\hline 6 & 6,62 & 5,83 & 5,61 \\
\hline
\end{tabular}

Sendo, NS: Nível de significância.

Médias seguidas de letras diferentes (maiúsculas nas colunas e minúsculas na linha) diferem entre si $(\mathrm{P} \leq 0,05)$ pelo teste de Tukey.

Com relação aos tempos de análise desses mesmos parâmetros $(1,5$, quatro e 24 horas postmortem), houve diferenças significativas $(\mathrm{P} \leq 0,05)$ entre os tempos de análises, e os maiores valores foram observados quando se mensurou o $\mathrm{pH}$ com 1,5 horas post-mortem $(6,60)$, quando comparados com os demais tempos de quatro e 24 horas $(5,79$ e 5,70, respectivamente), que também diferiram estatisticamente entre si $(\mathrm{P} \leq 0,05)$. Esses resultados já eram esperados, pois, após o abate dos animais, uma série de alterações ocorre durante a conversão do músculo em carne. Logo após o abate, devido à existência de reservas de glicogênio muscular e, portanto de ATP, o músculo mantém capacidade de contrair e relaxar. Durante esse período, que é de menos de 30 minutos em frangos, o glicogênio é convertido em ácido lático, reduzindo o $\mathrm{pH}$ original, que é de aproximadamente 7,2 para 5,7 a 5,9, quando este se estabiliza (SOUZA, 2006). 
Para o parâmetro valor $\mathrm{R}$, não foram encontradas diferenças significativas $(\mathrm{P}>0,05)$ entre as coletas. No entanto, houve diferenças significativas $(\mathrm{P} \leq 0,05)$ entre os tempos de análises para o valor de $\mathrm{R}$, e a menor média foi obtida quando se avaliou o valor $\mathrm{R}$ com 1,5 horas $(1,07)$ em comparação com os demais tempos de quatro e 24 horas post-mortem (1,29 e 1,43, respectivamente), que diferiram entre si $(\mathrm{P} \leq 0,05)$. Esses resultados expressam as mudanças bioquímicas que ocorrem no músculo durante a instalação do rigor mortis, traduzido pelo consumo das reservas de energia (o ATP) e, como consequência, a formação de AMP durante a contração muscular, resultando em aumento da relação AMP/ATP com o aumento do tempo postmortem. SCATOLINI et al. (2006) também observaram, em seu estudo acerca do efeito do tempo de desossa e tempo de armazenagem sobre a qualidade da carne de frango, aumento do valor $\mathrm{R}$ conforme o aumento do tempo post-mortem.

Houve interação significativa $(\mathrm{P} \leq 0,05)$ entre a coleta e o tempo de análise do $\mathrm{pH}$, e nas coletas 2, 4 e 6 foram encontrados os maiores valores quando se analisou o pH com 1,5 horas (6,55, 6,74 e 6,35, respectivamente), em comparação com os demais tempos de quatro e 24 horas post-mortem de análises que não diferiram entre si $(\mathrm{P}>0,05)$. Esses resultados já eram esperados, pois, com 1,5 horas post-mortem, ainda não houve a resolução do rigor mortis, portanto o pH ainda se encontra alto. Já com quatro e 24 horas postmortem, o pH se encontra estabilizado, mostrado pela não diferença significativa entre os dois tempos de análise, indicando a resolução do rigor mortis.

Na tabela 2, são mostrados os resultados da estatística descritiva para os parâmetros de qualidade da carne de peito de matrizes de descarte. Essa carne apresentou valores médios, mínimos e máximos do parâmetro valor de $L^{*}$ de 50,11, 41,38 e 59,59, respectivamente. Em comparação com a carne de peito de frango, a carne de matrizes de descarte possui valores de $\mathrm{L}^{*}$ superiores aos valores da carne de frango. KOMIYAMA (2006), em seus estudos acerca de caracterização da carne pálida em frangos de corte, relatou que filés de coloração normal apresentaram média em torno de 47,25. Os valores de a* (teor de vermelho) apresentaram valores médios de 3,16, valores mínimo e máximo de 0,52 e 8,70, respectivamente. O valor de b* médio da carne de matrizes é de 0,51 , com valores mínimo e máximo de -3,59 e 4,69, respectivamente. Já BORBA (2008), estudando o efeito da maturação na carne de matrizes de descarte, encontrou valores médios de valor L*, a* e b* de 49,08, 2,70 e 0,91, respectivamente, para carne não maturada.

Para a capacidade de retenção de água, os valores médio, mínimo e máximo da carne de peito foram de 76,67, 61,90 e 85,00\%, respectivamente. Esses valores estão de acordo com TAKAHASHI (2007), que avaliou a CRA da carne de peito de frangos de corte de três linhagens diferentes e observou valores de 72,13, 71,73 e 71,41\% para as linhagens Avian 48, Cobb e Hubbard, respectivamente. Foi analisada também a capacidade de absorção de água (CAA), que, pela estatística descritiva, apresentou valor médio de 57,74\%. Esses resultados indicam que a carne de matriz apresenta alta capacidade de absorção de água em comparação ao frango de corte, pois, segundo CAÑIZARES (2008), estudando o efeito da irradiação em carne de frangos de corte, encontrou valores de 39,70 e 30,18\% para a carne não irradiada e irradiada com 3kGy, respectivamente.

A carne de peito de matrizes de descarte apresentou porcentagem de perda de exsudato de $2 \%$, com valores mínimo e máximo de 1,13 e 3,67\%,

Tabela 2 - Estatística descritiva dos parâmetros de qualidade da carne de matrizes de descarte.

\begin{tabular}{|c|c|c|c|c|c|}
\hline Qualidade & Média & Mínimo & Máximo & Desvio padrão & $\mathrm{CV}$ \\
\hline $\mathrm{L}^{*}$ & 50,11 & 41,38 & 59,59 & 3,27 & 6,53 \\
\hline$a^{*}$ & 3,16 & 0,52 & 8,70 & 1,35 & 42,76 \\
\hline$b^{*}$ & 0,51 & $-3,59$ & 4,69 & 1,66 & 326,44 \\
\hline CRA (\%) & 76,67 & 61,90 & 85,00 & 5,05 & 7,04 \\
\hline CAA (\%) & 57,75 & 24,57 & 98,86 & 16,74 & 28,99 \\
\hline PE (\%) & 2,00 & 1,13 & 3,67 & 0,49 & 24,59 \\
\hline $\mathrm{CE}\left(\mathrm{ml} 0,5 \mathrm{~g}^{-1}\right)$ & 70,22 & 41,4 & 98,8 & 12,33 & 17,56 \\
\hline PPC (\%) & 17,92 & 4,31 & 36,18 & 5,98 & 33,38 \\
\hline FC $\left(\mathrm{Kgf} \mathrm{cm}^{-2}\right)$ & 4,94 & 2,37 & 10,94 & 1,88 & 38,12 \\
\hline
\end{tabular}

L*: Teor de luminosidade; a*: teor de vermelho; b*: teor de amarelo; CRA: Capacidade de retenção de água; CAA: Capacidade de absorção de água; PE: Perda de exsudato; CE: Capacidade de Emulsificação; PPC: Perda de peso por cozimento; FC: Força de cisalhamento; CV: Coeficiente de variação. 
respectivamente. Esses dados se assemelham aos relatados por OLIVO (1999), o qual relatou que os grupos de aves não estressadas (controle) apresentaram teor de exsudato de 1,70. A perda de exsudato, que é a consequência do encolhimento postmortem das miofibrilas em razão da diminuição do $\mathrm{pH}$, é uma das principais causas da diminuição da qualidade dos produtos cárneos nas indústrias (JENSEN et al., 1998). Para a capacidade de emulsificação, foram obtidos valores médios de $70,22 \mathrm{ml}_{0,5 \mathrm{~g}^{-1}}$. Esses resultados estão ligeiramente abaixo dos resultados citados por NUNES (2003), que encontrou valores médios para esse parâmetro de $75 \mathrm{ml} 0,5 \mathrm{~g}^{-1} \mathrm{em}$ carne de peito de matrizes de descarte. Assim, a carne de matrizes de descarte apresenta boa capacidade de formar emulsões, podendo ser utilizada como matéria-prima na elaboração de produtos emulsionados.

A perda de peso média por cozimento dessa carne foi de 17,92\%. A carne apresentou valores mínimo e máximo de 4,31 e 36,18\%. Porém, OLIVO (1999) encontrou valores para perda de peso por cozimento entre grupos de aves estressadas e calmas em frangos de corte variando de 11,26 a $12,01 \%$, sendo possivelmente explicados por uma PPC relativamente baixa, considerando-se que a quebra de peso de um filé de frango ou de outro corte cárneo normalmente se encontra em torno de $30 \%$ durante o seu cozimento (fritura ou grelha). Pela estatística descritiva, foi observada média de força de cisalhamento de 4,94kgf $\mathrm{cm}^{-2}$. Quando se compara o peito de matriz com peito de frangos de corte, pode-se constatar que a carne de matriz apresenta-se ligeiramente menos macia que a carne de frango, o que pode ser observado em inúmeros trabalhos na literatura. CAÑIZARES (2008) observou valores de força de cisalhamento de $2,43 \mathrm{kgf}^{-2}$ para filés de frango não irradiados. Valores semelhantes foram encontrados por KOMIYAMA et al. (2008), os quais, estudando o efeito do tempo de jejum sobre a qualidade da carne de frangos de corte, encontraram valores de 2,28; 2,94; 2,98 e 2,96kgf $\mathrm{cm}^{-2}$ para os tratamentos de quatro, oito, 12 e 16 horas de jejum ante-mortem. No entanto, BORBA (2008), em seus estudos sobre o efeito da maturação na carne de matrizes de descarte, observou valores de FC de 4,50 e $3,56 \mathrm{kgf} \mathrm{cm}^{-2}$ para a carne não maturada e maturada por dois dias. Assim, os resultados desse autor se assemelham aos encontrados neste trabalho, permitindo afirmar que a carne de matriz apresenta FC aumentada em comparação à carne de frangos de corte.

Para as características sensoriais da carne de peito de matrizes, houve diferenças significativas $(\mathrm{P} \leq 0,05)$ para os parâmetros intensidade de aroma, sabor, sabor estranho, maciez, suculência e mastigabilidade entre os tipos de carne analisados (Tabela 3). A intensidade de aroma característico de carne de frango foi de 6,4 e 5,8 para a carne de frango e carne de matriz, respectivamente. Em termos quantitativos, isso mostra que os provadores verificaram aroma menos característico de carne de frango na carne de matriz. Porém, a carne de matrizes apresenta, pela escala, variação entre aroma moderadamente fraco e moderadamente forte, enquanto para a carne de frango a variação ficou entre moderadamente forte e forte, não apresentando grandes diferenças de aroma. Não foram encontradas diferenças significativas $(\mathrm{P}>0,05)$ para o aroma estranho entre a carne de matriz e a frango. Esse resultado permite concluir que a carne de matriz não apresenta alteração no aroma, como foi citado por ROUSELLE et al. (1984), os quais relataram que a intensidade do aroma poderia

Tabela 3 - Análise sensorial da carne de peito de matrizes pesadas de descarte comparado com a carne de peito de frangos de corte.

\begin{tabular}{|c|c|c|c|c|c|}
\hline Característica & Matriz & DP & Frango & $\mathrm{DP}$ & NS \\
\hline Intensidade de aroma $^{1}$ & 5,8 & 1,80 & 6,4 & 1,68 & 0,0411 \\
\hline Aroma estranho ${ }^{2}$ & 1,6 & 1,32 & 1,1 & 0,45 & 0,1110 \\
\hline Sabor $^{3}$ & 6,5 & 1,41 & 7,3 & 0,87 & 0,0066 \\
\hline Sabor estranho ${ }^{4}$ & 1,9 & 1,38 & 1,2 & 0,66 & 0,0270 \\
\hline Maciez $^{5}$ & 6,9 & 0,83 & 2,5 & 1,44 & $<, 0001$ \\
\hline Suculência ${ }^{6}$ & 3,4 & 1,25 & 6,5 & 1,32 & $<, 0001$ \\
\hline Mastigabilidade $^{7}$ & 2,7 & 1,56 & 6,9 & 1,39 & $<, 0001$ \\
\hline
\end{tabular}

${ }^{1}$ Escala variando do sem aroma ao muito intenso e característico; ${ }^{2}$ 1-nenhum, 2-extremamente fraco, 3-muito franco, 4-fraco, 5moderadamente fraco, 6-moderadamente forte, 7-forte, 8-muito forte, 9-extremamente forte; ${ }^{3}$ Escala variando do muito ruim ao muito bom; 4 1-nenhum, 2-extremamente fraco, 3-muito franco, 4-fraco, 5-moderadamente fraco, 6-moderadamente forte, 7-forte, 8-muito forte, 9extremamente forte; ${ }^{5}$ 1-extremamente macio (catupiri), 2-muito macia, 3-moderadamente macia, 4-macia, 5-nem macia nem dura (azeitona), 6-levemente dura, 7-moderadamente dura, 8-muito dura, 9-extremamente dura (bala soft); ${ }^{6}$ 1-extremamente seco, 2-muito seco, 3moderadamente seco, 4-levemente seco, 5- nem seco nem suculento, 6-levemente suculento, 7-moderadamente suculento, 8-muito suculento, 9- extremamente suculento; ${ }^{7}$ Escala variando de elástica, borrachenta, difícil de deglutir ao desintegrar facilmente na boca, fácil de deglutir. Sendo: DP: Desvio Padrão e NF: Nível de significância. 
ser um problema para esse tipo de carne por ser proveniente de animais mais velhos.

Para os provadores, a carne de matriz apresentou menor intensidade $(6,5)$ de sabor quando comparada com a carne de frango $(7,3)$. Além disso, apresentou maior intensidade de sabor estranho $(1,9)$, em comparação com a carne de frango $(1,2)$, o que, segundo ROÇA (1986), pode ser atribuído à diferença de paladar entre provadores, pois os valores observados são muito próximos. A carne de peito de matriz apresentou baixa intensidade de maciez $(6,9)$ quando comparada com a carne de frango $(2,5)$. Esses dados corroboram ROLAND et al. (1981), que relataram problemas com relação à textura, visto que animais mais velhos apresentam carne notadamente mais dura. A carne de matriz apresentou menor suculência $(3,4)$, quando comparada à carne de peito de frangos $(6,5)$. Quando se avaliou a mastigabilidade, a carne de peito de matriz apresentou-se mais elástica, borrachenta e difícil de deglutição $(2,7)$ do que a carne de peito de frango, que apresentou como características a fácil desintegração na boca e facilidade de deglutição $(6,9)$.

\section{CONCLUSÃO}

A carne de matrizes pesadas de descarte apresenta boas características de qualidade tecnológica que possibilitam sua utilização como matéria-prima para a elaboração de industrializados. No entanto, apresentou problema em alguns atributos sensoriais, especialmente a maciez, suculência e mastigabilidade, em comparação com a carne de peito de frango. Desse modo, como forma de melhorar essas características sensoriais, devem ser utilizados processamentos tecnológicos adequados que possibilitem sua comercialização e a satisfação do consumidor final.

\section{FONTES DE AQUISIÇÃO}

O trabalho foi financiado pelo Conselho Nacional de Desenvolvimento Científico e Tecnológico (CNPq), na forma de bolsa de doutorado para a primeira autora, e pela FAPESP, na forma de suporte financeiro.

\section{REFERÊNCIAS}

AMERICAN MEAT SCIENCE ASSOCIATION (AMSA). Research guideliness for cookery sensory and instrumental tenderness measurement of fresh meat. Chicago, 1995. 48p.

SPLITTSTOESSER, D.F. Compendium of methods for the examination of foods. 3.ed. Washington: APHA, 1992. 1219p.
BENDALL, J.R. Post-mortem change in muscle. In: BOURNE, G.H. (Ed.). The structure and function of muscle. New York. Academic, 1973. p.242-309.

BORBA, H. Utilização do processo de maturação e marinação sobre as características qualitativas da carne de matrizes de descarte de corte e galinhas poedeiras de descarte. 2008. 50f. Tese (Livre Docência) - Faculdade de Ciências Agrárias e Veterinárias - UNESP-Campus de Jaboticabal, SP.

CAÑIZARES, M.C. Qualidade da carne de frango submetida a irradiação ou atmosfera modificada e armazenada por diferentes períodos. 2008. 105f. Tese (Doutorado em Zootecnia) - Curso de Pós-graduação em Zootecnia, Faculdade de Medicina Veterinária e Zootecnia, Universidade Estadual Paulista, Botucatu, SP.

HAMM, R. Biochemistry of meat hydratation. Advances in Food Research, Cleveland, v.10, n.2, p.335-443, 1960.

HONIKEL, K.O.; FISHER C.A. A rapid method for the detection of PSE and DFD porcine muscles. Journal of Food Science, Chicago, Illinois, v.42, n.7, p.1663-1676, 1977.

HONIKEL KO. Influence of chilling on meat quality attributes of fast glycolysing pork muscles. In: TARRANT, P.V. et al. (Eds.). Evaluation and control of meat quality in pigs. Dordrecht: Martinius Nijhoff, 1987. p.273-283.

JENSEN, C. et al. Dietary vitamin E: quality and storage stability of pork and poultry. Trends Food Science Technology, Cambridge, v.9, p.62-72, 1998.

KOMIYAMA, C.M. Caracterização e ocorrência de carne pálida em frangos de corte e seu efeito na elaboração de produtos industrializados. 2006. 89f. Dissertação (Mestrado em Zootecnia) - Curso de Pós-graduação em Zootecnia, Faculdade de Medicina Veterinária e Zootecnia, Universidade Estadual Paulista, Botucatu, SP.

KOMIYAMA, C.M. et al. Chicken meat quality as a function of fasting period and water spray. Revista Brasileira de Ciência Avícola / Brazilian Journal of Poultry Science, Campinas (SP), v.10, p.147-151, 2008. Disponível em: <http:/ /www.scielo.br/scielo.php?script=sci_arttext\&pid=S1516635X2008000300008>. Acesso em: 10 jan. 2009. doi: 10.1590/S1516-635X2008000300008.

NAKAMURA, R. et al. The contribuition of intramuscular collagen to the tenderness of meat from chicken with different ages. Poultry Science, Champaign (Ilinois), v.54, p.16041612, 1975.

NUNES, TP. Efeito da pré-cura na estabilidade microbiológica de carne mecanicamente separada e elaboração de carne mecanicamente separada e elaboração de um produto reestruturado com filés de peito de galinhas de descarte. 2003. 101f. Dissertação (Mestrado em Ciência e Tecnologia de Alimentos) Departamento de Agroindústria, Alimentos e Nutrição, Escola superior de Agricultura Luiz de Queiroz, Piracicaba, SP.

NURMAHMUDI, A.R.; SAMS, A.R. Tenderizing spent fowl meat with calcium chloride. 1. Effect of delivery method and tumbling. Poultry Science, Champaign (Illinois), v.76, p.534-537, 1997.

Ciência Rural, v.40, n.7, jul, 2010. 
OIE - Organização Internacional de Epzootias. 2002. Disponível em: <http://www.oie.int/eng/normes/mcode/ A_summry.htm>. Acesso em: 28 de maio, 2009.

OLIVO R. Carne PSE em frangos. 1999. 97f. Tese (Doutorado em Ciência dos Alimentos) - Faculdade de Ciências Farmacêuticas, Universidade de São Paulo, São Paulo, SP.

RASMUSSEN, A.; ANDERSSON, M. New methods for determination of drip loss in pork muscles. In: INTERNATIONAL CONGRESS OF MEAT SCIENCE AND TECHNOLOGY, LILlEHAMMER, 42., 1996, Norway. Proceedings... Norway: HILDRUM \& RISVIK (Ed.),1996. p.286-287.

ROÇA R.O. Desenvolvimento de fiambres com carne de frango. 1986. 183f. Dissertação (Mestrado em Engenharia de Alimentos, Área de Tecnologia de Alimentos) - Faculdade de Engenharia de Alimentos, Universidade Estadual de Campinas, SP.

ROÇA, R.O.; BONASSI, I.A. Seleção de provadores para produtos cárneos. In: CONGRESSO BRASILEIRO DE CIÊNCIA E TECNOLOGIA DE ALIMENTOS, 7., 1985, Itabuna/Ilhéus. Anais... Itabuna/Ilhéus, SBCTA, 1985. p.83.

ROÇA, R.O. et al. Utilização de toucinho na elaboração de fiambres com carne de frango. Ciência e Tecnologia de Alimentos, Campinas, v.8 n.1, p.67-76, 1988.

ROLAND, L.M. et al. Physical and sensorial proprieties of chicken patties made with varying proportions of white and dark spent fowl meat. Journal of Food Science, Chicago, Illinois, v.46, n.3, p.834-837, 1981.
ROUSELLE, J.R. et al. Utilizing flake-cut spent fowl meat in chicken patties. Poultry Science, Champaign (Illinois), v.63, n.5, p.932-937, 1984.

SAS Institute. SAS Users guide. Cary, Nc, 1998. 176p.

SCATOLINI, A.M. et al. Efeito do período de desossa e do armazenamento em refrigeração sobre a aqualidade da carne de peitos de frango. Revista Portuguesa de Ciências Veterinárias, Lisboa, v.101, p.(559-560) 257-262, 2006. Disponível em: http://www.fmv.utl.pt/spcv/PDF/pdf12_2006/ 257-262.pdf. Acesso em: 15 nov. 2008.

SOUZA, H.B.A. Parâmetro físicos e sensoriais utilizados para avaliação da qualidade da carne de frango. In: SEMINÁRIO DE AVES E SUÍNOS - AVESUI, 2006, Florianopolis. Anais... São Paulo: Gessuli Agribusiness, 2006. p.91-96.

SWIFT, C.E. et al. Comminuted meat emulsions: the capacity of mat for emulsifying fat. Food Technology, Chicago, Illinois. v.15, n.11, p.468-473, 1961.

TAKAHASHI, S.E. Ocorrência de carne pálida e características de qualidade de carne de frangos de corte. 2007. 86f. Tese (doutorado em Zootecnia) - Departamento de Produção Animal, Faculdade de Medicina Veterinária e Zootecnia, Universidade Estadual Paulista, Botucatu, SP.

UBA - UNIÃO BRASILEIRA DE AVICULTURA. Alojamento de matrizes para corte. Relatório Anual 2007/2008. São Paulo, 2009. p.37-38.

VAN LAACK, R.L.J.M. et al. Characteristics of pale, soft, exudative broiler breast meat. Poultry Science, Champaign (Illinois) v.79, n.7, p.1057-1061, 2000 